\title{
Actualización del PLC de un Sistema de Manufactura Integrada por Computador
}

\author{
Ivone Cecilia Torres Rodríguez ${ }^{1}$, Pedro Francisco Huerta González¹, Zosimo Ismael \\ Bautista Bautista ${ }^{1}$.
}

ictorres@ipn.mx,phuerta@ipn.mx, zbautista@ipn.mx.

${ }^{1}$ Instituto Politécnico Nacional, ESIME Zacatenco, Departamento Académico de Ingeniería en Control y Automatización. Av. IPN S/N, Col. La Escalera, C.P 07820, Ciudad de México, México.

DOI: $10.17013 /$ risti.27.18-35

\begin{abstract}
Resumen: En el presente artículo se detalla la migración y rehabilitación de la etapa de control de un Sistema de Manufactura Integrada por Computador, mediante la implementación de un sistema de automatización actual para el uso didáctico y profesional de los alumnos de la carrera de Ingeniería en Control y Automatización. Se realiza la selección de los nuevos equipos de control para cada una de las estaciones de trabajo que la componen, se desarrolla la topología de interconexión entre las diferentes estaciones mediante el protocolo EtherNet/IP, y se diseña e implementa la lógica de funcionamiento de las estaciones.
\end{abstract}

Palabras-clave: CIM; EtherNet/IP; PLC; Protocolo

\section{PLC Update of a Computer-Integrated Manufacturing System}

Abstract: In this article the migration and rehabilitation of the control stage of a Computer Integrated Manufacturing System is detailed, through the implementation of a recent automation system for the didactic and professional use of the students of the Control Engineering and Automation. The selection of the new control equipment for each of the work stations that compose it is carried out, the interconnection topology is developed between the different stations through the EtherNet/IP protocol, the operating logic of the stations is designed and implemented.

Keywords: CIM; EtherNet/IP; PLC; Protocol

\section{Introducción}

Debido al constante movimiento y evolución de la tecnología dentro del sector productivo, las industrias enfrentan una intensa competencia por mantener presencia en el mercado, de modo que deben asumir nuevas y mejores estrategias de producción que respondan de manera efectiva al cambio rápido y constante; buscando utilizar tecnología que incremente la capacidad de manufacturar piezas, o productos terminados, usando 
procesos y estrategias de manufactura flexible y capaces de modificarse, adaptándose a los nuevos requerimientos, por ello hoy en día se libra una intensa batalla por mantenerse a la vanguardia (Kalpakjian \& Schmid, 2001),( Radhakrishnan, Subramanyan, \& Raju, 2000). Aquella tecnología que no es capaz de innovarse a sí misma es condenada a la desaparición por obsolescencia (Garzón, 2001).

En esta carrera tecnológica tan veloz, las instituciones educativas no pueden limitarse a ser testigos pasivos de lo que ocurre, sino por el contrario, deberán ser fuertes generadoras, impulsores y promotoras del progreso generado en el mercado.

Aun cuando resulte difícil conseguirlo por limitantes de infraestructura o recursos, las alternativas podrán verse siempre nacidas de trabajos como éste, para acotar las distancias entre los despuntes tecnológicos y el conocimiento didáctico.

El presente trabajo muestra la migración de un Sistema Integral de Manufactura que se encuentra en la Escuela Superior de Ingeniería Mecánica y Eléctrica (ESIME) Unidad Zacatenco, cuya estructura está conformada con tres estaciones que en su conjunto simulan procesos CIM, utilizados a nivel industrial. El sistema se encontraba en un estado de obsolescencia tecnológica, además del desgaste evidente por el paso de los años y el uso de anteriores generaciones, por lo cual fue necesaria su actualización para ser aplicado a las unidades de aprendizaje de la carrera de Ingeniería en Control y Automatización y así contribuir en el aprendizaje a los alumnos en esta disciplina.

\section{Marco teórico}

El acrónimo CIM es ampliamente conocido en la Industria, pero fue hasta el año 1973 cuando fue introducido por primera vez por Harrington. $\mathrm{J}$ en su libro llamado Computer Integrated Manufacturing (Manufactura Integrada por Computadora) (García \& Castillo, 2007).

Si bien varios autores definen al CIM de distinta manera, tienen en común mencionar que el CIM busca integrar todas las actividades de la empresa por medio de la tecnología que ofrecen las computadoras (Tejada, 2001); esta estrategia de producción busca unificar la información de las distintas áreas para la gestión y control de los procesos; así se incorporan todos los pasos de un proceso de manufactura, esta integración permite que todos los procesos individuales se comuniquen entre sí de manera rápida e inicien acciones, con ello la fabricación puede ser rápida y menos propensa a errores o posibles contratiempos, por otro lado, poseen flexibilidad de diseño por lo que pueden ser ajustados de manera adecuada. Dentro del CIM se cubren puntos como diseño, análisis, planeación, costos, logística, almacenamiento, gestión y distribución de productos para proveer un monitoreo y control de todas las operaciones (Elanchezhian, Sunder, \& Shanmuga, 2007).

En lo concerniente a la estructura del CIM desafortunadamente no existe una única descomposición de cada uno de los eslabones que lo conforman. Cada industria puede definir tantos subsistemas y partes que desee, de acuerdo a sus requerimientos (Grover \& Zimmers, 1984) (Porras, 2003). Así pues, debetomarse en cuenta quelas industrias realizan procesos ajenos entre sí, y aunque también hay industrias que realizan el mismo proceso, estas siempre tendrán diferentes necesidades del cliente y diseño del producto, de modo 
que, aunque posean sistemas CIM, estos difícilmente poseerán la misma estructura. Un intento por mostrar las partes que conforman el CIM es muestra en la figura 1.

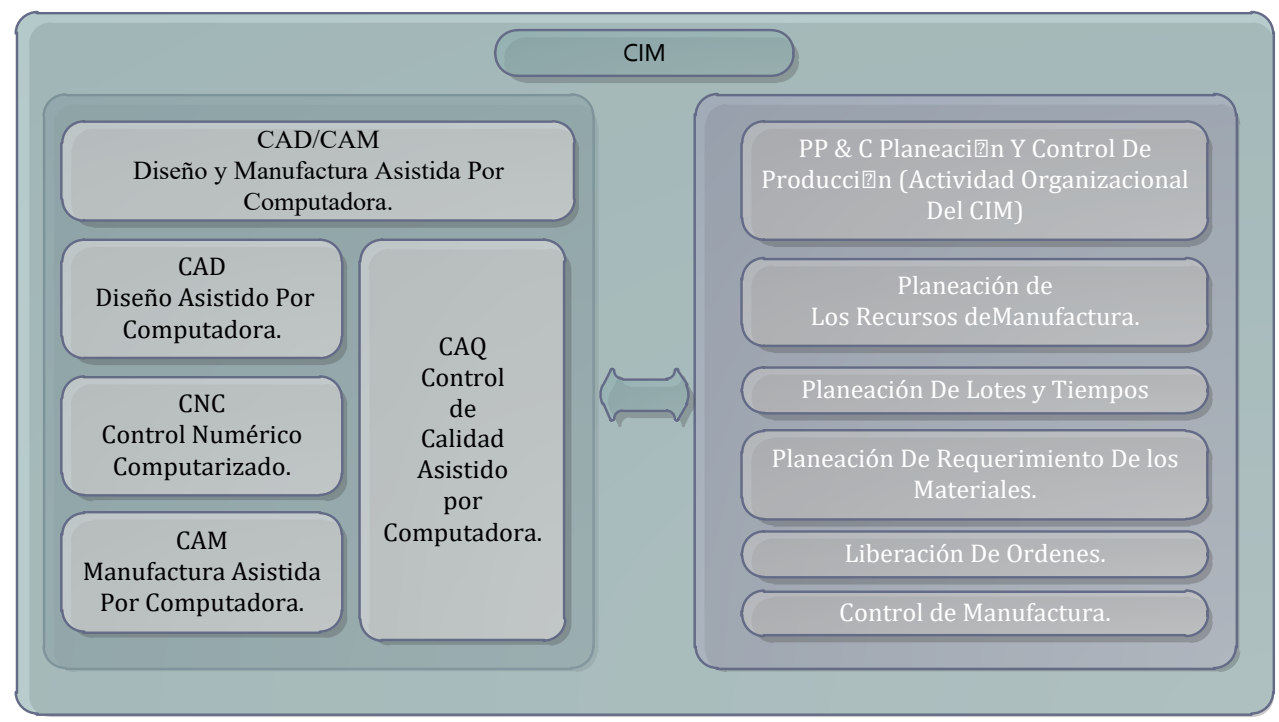

Figura 1 - Estructura del CIM

\subsection{CIM-2000}

La Escuela Superior de Ingeniería Mecánica y Eléctrica cuenta con el modelo CIM-200o Mechatronics (System, 1994), este equipo sirve como herramienta de entrenamiento



Figura 2 - Estaciones del CIM. 
para introducir a los alumnos a un ambiente industrial que servirá al proceso de su formación profesional; de modo que sea posible acercarse a las metodologías, conceptos, herramientas, técnicas e instalaciones que conciernen propiamente al modelo CIM y procesos de manufactura industriales.

El CIM está conformado por las estaciones de: control central, almacenamiento y la estación neumática, las cuales se muestran en la figura 2.

\subsection{Descripción de la estación de control}

La estación de control central es la responsable de coordinar el comportamiento de cada una de las estaciones del CIM, estaba formada por tres Controladores Lógicos Programables (PLC) de la familia Modicon Compact 984 ya descontinuados, y dos computadoras para la programación y monitoreo del sistema (System, 1994).

En esta estación, se realizaban las funciones de ajuste y programación de las estaciones de trabajo, se efectuaba el monitoreo, supervisión y gestión del orden de trabajo, el arranque y paro de las mismas y la banda transportadora, y el monitoreo de los pallets que se transportaban. Su inconveniente era que al mismo tiempo se utilizaba para el monitoreo y manejo de la planta, la carencia de funciones para la supervisión de los datos, la inexistencia de un módulo para aplicar algoritmos del proceso y mantener estos valores dentro de ciertos límites.

\subsection{Descripción de la estación neumática}

Esta estación es la encarga de alimentar el sistema de manufactura (figura 3), con base en una orden preparada en la estación central de control CIM-2000, esta área no es la responsable del funcionamiento directo sobre ella, solo es responsable de coordinar el orden de operación entre las estaciones (System, 1994).



Figura 3 - Estación neumática.

Para comenzar su operación, la estación de control detecta la señal, que un pallet está en el puerto de carga y descarga de la estación neumática, en ese momento, el actuador de paro se activa deteniendo el avance del mismo, a partir de allí la estación neumática es responsable del comportamiento de los elementos que la componen. Esta estación, está equipada con los siguientes dispositivos: 
- Contenedor de vagones. Incluye un actuador neumático y sus sensores.

- Contenedor de prismas de acrílico. Contiene un cilindro neumático equipado con un final de carrera mecánico.

- Contenedor de cilindros de aluminio. Contiene 5 actuadores neumáticos y sensores.

- Brazo Neumático I. Conformado por 4 actuadores neumático y sensores; transporta los cilindros del contenedor de cilindros al de vagones.

- Brazo Neumático II. Compuesto por 4 actuadores y sensores; se cargar y descargar los vagones estacionados en la estación neumática.

\subsection{Descripción de la estación de almacenamiento}

Esta estación ubica productos terminados y material no trabajado en sus celdas (System, 1994). Si en algún momento un elemento es llamado a una estación del sistema, y si la estación no está lista, el vagón es almacenado en la celda más cercana y disponible de la estación de almacenamiento (figura 4).

Cuando la estación del proceso a realizar vuelve a estar disponible, esta avisa a la estación central de control, para mandar una instrucción al almacén y liberar el elemento almacenado. Esta estación está equipada con los siguientes dispositivos:

- 32 celdas de almacenamiento, en una matriz de 8 columnas y 4 filas.

- Un manipulador cartesiano que dispone de 2 motores de CD para desplazarse en los ejes X y Y, para almacenar los vagones o piezas.

- Un cilindro neumático de doble efecto para cargar y descargar los vagones.



Figura 4 - Estación de almacenamiento.

\section{Desarrollo de la Metodología}

Para la rehabilitación del sistema, se realizó la selección de los Controladores Lógicos Programables, módulos de entradas y salidas requeridas, y las Fuentes de alimentación para los dispositivos involucrados en el funcionamiento del sistema.

El PLC seleccionado para la estación de control fue el CompactLogix 5370 L3 (Automation, 2012), y para las estaciones neumática y de almacenamiento se seleccionaron 
los CompactLogix 5370 L2 (Automation, 2013), todos de la marca Allen Bradley. Los módulos de entrada y salida seleccionados para cada uno de los controladores elegidos fueron 1769 Compact I/O.

A continuación, se realizan los cálculos necesarios para la selección de las fuentes de alimentación por estación. En esta sección se muestran los datos, consideraciones y cálculos necesarios para la adecuada selección de la fuente de alimentación, partiendo de los dispositivos que se conectarán a los módulos de E/S 1769; tales como válvulas solenoides, contactos para el control de motores, dispositivos de indicación como lámparas, sensores inductivos, capacitivos, reed switch, etc.

\subsection{Cálculos de Corriente de Entrada y Salida de la Estación de Control CIM-20oo}

Corriente de Entrada: Para el cálculo de la corriente total de optoacopladores $\left(I_{T O}\right)$ con relación al módulo de entradas digitales 1769-IQ32 (Automation, 2015), se calcula mediante la Ecuación III.1 en donde la corriente de cada optoacoplador $\left(I_{O}\right)$ es de $2 \mathrm{~mA}$.

$$
\begin{gathered}
I_{\text {TO }}=16 I_{O} \\
I_{\text {TO }}=16(2 \mathrm{~mA})=32 \mathrm{~mA}
\end{gathered}
$$

Corriente de Salida: La estación de control dispone de luces indicadoras en donde cada una consume una corriente $\left(I_{L}\right)$ de $240 \mathrm{~mA}$, así como solenoides que consumen una corriente cada una $\left(I_{S}\right)$ de $187.5 \mathrm{~mA}$, los cuales se conectan al módulo de salidas digitales 1769-OB16 (Automation, 2015), por lo tanto se consideran las Ecuaciones III.2 y III.3 para obtener la corriente total de luces indicadoras $\left(I_{T L}\right)$ y la corriente total de las solenoides $\left(I_{T S}\right)$ respectivamente.

$$
\begin{gathered}
I_{T L}=4 I_{L} \\
I_{T L}=4(240 \mathrm{~mA})=0.96 \mathrm{~A} \\
I_{T S}=4 I_{S} \\
I_{T S}=4(187.5 \mathrm{~mA})=0.75 \mathrm{~A}
\end{gathered}
$$

De modo que la corriente total de salida $\left(I_{S S}\right)$ se calcula con la Ecuación III.4

$$
I_{S S}=I_{T L}+I_{T S}=0.96 \mathrm{~A}+0.75 \mathrm{~A}=1.71 \mathrm{~A}
$$




\subsection{Cálculos de Corriente de Entrada y Salida de la Estación Neumática PN-280o}

Corriente de Entrada: Para el cálculo de la corriente total de optoacopladores $\left(I_{T O}\right)$ con relación al módulo de entradas digitales 1769-IQ32 (Automation, 2015), se calcula mediante las Ecuaciones III.5 en donde la corriente de cada optoacoplador $\left(I_{O}\right)$ es de 2 $\mathrm{mA}$, la III.6 en donde la corriente de cada sensor inductivo $\left(I_{I}\right)$ es de $200 \mathrm{~mA}$ y la III.7 en donde la corriente de cada sensor reed switch $\left(I_{S}\right)$ es de $300 \mathrm{~mA}$.

$$
\begin{gathered}
I_{T O}=22 I_{O} \\
I_{T O}=22(2 \mathrm{~mA})=44 \mathrm{~mA} \\
I_{S I}=5 I_{l} \\
I_{S I}=5(200 \mathrm{~mA})=1 \mathrm{~A} \\
I_{R S}=2 I_{S} \\
I_{R S}=2(300 \mathrm{~mA})=600 \mathrm{~mA}
\end{gathered}
$$

De modo que la corriente total de las entradas $\left(I_{E E}\right)$ se encuentra mediante la Ecuación III.8, en donde $I_{T O}$ es la corriente total de optoacopladores, $I_{S I}$ es la corriente total de los sensores inductivos e $I_{R S}$ es la corriente total de los sensores reed switch.

$$
l_{E E}=I_{T O}+I_{S I}+I_{R S}=0.044 \mathrm{~A}+1 \mathrm{~A}+0.6 \mathrm{~mA}=1.644 \mathrm{~A}
$$

Corriente de Salida: En la presente estación solo intervienen 15 válvulas solenoides en donde cada una consume una corriente $\left(I_{S}\right)$ de $187.5 \mathrm{~mA}$ y 4 lámparas indicadoras que consumen una corriente cada una $\left(I_{L}\right)$ de $240 \mathrm{~mA}$ conectadas al módulo de salidas digitales incorporado en el PAC 1769-L27ERM-QBFC1B (Automation, 2013). Por lo tanto, se consideran las Ecuaciones III.9 y III.10 para obtener la corriente total de las solenoides $\left(I_{T S}\right)$ y la corriente total de luces indicadoras $\left(I_{T L}\right)$ respectivamente.

$$
\begin{gathered}
I_{T S}=15 I_{S} \\
I_{T S}=15(187.5 \mathrm{~mA})=2.812 \mathrm{~A} \\
I_{T L}=4 I_{L}
\end{gathered}
$$




$$
I_{T L}=4(240 \mathrm{~mA})=0.96 \mathrm{~A}
$$

De modo que la corriente total de salida $\left(I_{S S}\right)$ se calcula con la Ecuación III.11

$$
I_{S S}=I_{T S}+I_{T L}=2.812 \mathrm{~A}+0.96 \mathrm{~A}=3.772 \mathrm{~A}
$$

\subsection{Cálculos de Corriente de Entrada y Salida de la Estación de Almacenamiento ST-200o}

Corriente de Entrada: Para el cálculo de la corriente total de optoacopladores $\left(I_{T O}\right)$ en relación a los módulo de entradas digitales 1769-IQ16F (Automation, 2015) y el módulo de entradas digitales incluido en el PAC 1769-L27ERM-QBFC1B, se calcula mediante las Ecuaciones III.12 en donde la corriente de cada optoacoplador $\left(I_{O}\right)$ es de $2 \mathrm{~mA}$, la III.13 en donde la corriente de cada sensor inductivo $\left(I_{P}\right)$ es de $200 \mathrm{~mA}$ y la III.14 en donde la corriente de cada sensor reed switch $\left(I_{S}\right)$ es de $300 \mathrm{~mA}$.

$$
\begin{gathered}
I_{\text {TO }}=17 I_{O} \\
I_{T O}=17(2 \mathrm{~mA})=34 \mathrm{~mA} \\
I_{S I}=1 I_{I} \\
I_{S I}=1(200 \mathrm{~mA})=200 \mathrm{~mA} \\
I_{R S}=2 I_{S} \\
I_{R S}=2(300 \mathrm{~mA})=600 \mathrm{~mA}
\end{gathered}
$$

De modo que la corriente total de las entradas $\left(I_{E E}\right)$ se encuentra mediante la Ecuación III.15, en donde $I_{T O}$ es la corriente total de optoacopladores, $I_{S I}$ es la corriente total de los sensores inductivos e $I_{R S}$ es la corriente total de los sensores reed switch.

$$
I_{E E}=I_{T O}+I_{S I}+I_{R S}=34 \mathrm{~mA}+200 \mathrm{~mA}+600 \mathrm{~mA}=834 \mathrm{~mA}
$$

Corriente de Salida: En la presente estación los dispositivos se conectan al módulo de salidas digitales embebido del PAC 1769-L27ERM-QBFC1B, conectando 3 válvulas solenoides en donde cada una consume una corriente $\left(I_{S}\right)$ de $187.5 \mathrm{~mA}, 4$ lámparas indicadoras que consumen una corriente cada una $\left(I_{L}\right)$ de $240 \mathrm{~mA}$ y 4 bobinas de relevador en donde cada una consume una corriente $\left(I_{B}\right)$ de $260 \mathrm{~mA}$, cuyos contactos accionan a 2 motores de 8.60 A $\left(I_{M}\right)$. Por lo tanto, se consideran las Ecuaciones III.16, III.17, III.18 y III.19 para obtener la corriente total de las solenoides $\left(I_{T S}\right)$, la corriente total de luces indicadoras $\left(I_{T L}\right)$, la corriente total de bobinas $\left(I_{T B}\right)$ y la corriente total de los motores $\left(I_{T M}\right)$ respectivamente. 


$$
\begin{gathered}
I_{T S}=3 I_{S} \\
I_{T S}=3(187.5 \mathrm{~mA})=562 \mathrm{~mA} \\
I_{T L}=4 I_{L} \\
I_{T L}=4(240 \mathrm{~mA})=0.96 \mathrm{~A} \\
I_{T B}=4 I_{L} \\
I_{T B}=4(260 \mathrm{~mA})=1.04 \mathrm{~A} \\
I_{T M}=2 I_{M} \\
I_{T M}=2(8.60 \mathrm{~A})=17.2 \mathrm{~A}
\end{gathered}
$$

De modo que la corriente total de salida $\left(I_{S S}\right)$ se calcula con la Ecuación III.20

$$
\begin{gathered}
I_{S S}=I_{T S}+I_{T L}+I_{T B}+I_{T M} \\
I_{S S}=0.562 \mathrm{~A}+0.240 \mathrm{~A}+1.04 \mathrm{~A}+17.2 \mathrm{~A}=19.042 \mathrm{~A}
\end{gathered}
$$

Una vez que se seleccionaron los controladores, módulos E/S, fuentes de alimentación, se realizan los diagramas de conexión entre sensores y controladores, diagramas de fuerza y control, así como la conexión entre cada uno de los elementos.

\subsection{Diagrama de Conexiones}

En la figura 5 se muestra el diagrama de conexión eléctrica de la estación de control, se observa la fuente de alimentación del PLC, la alimentación hacia la interfaz gráfica y la alimentación hacia la fuente 1606-XLE8oE, encargada de alimentar a cualquier elemento que no sea el controlador de la estación. Por otro lado, en esta figura se observa un enclave, el cual tiene como objetivo habilitar y deshabilitar las señales que se dirigen desde y hacia el controlador de la estación. Al presionar el Botón de arranque (BA) la bobina MCR se energiza, y al instante queda enclavada por acción de su contacto normalmente abierto MCR1. Como resultado del enclave el contacto MCR2 normalmente abierto, se cierra el circuito energizando a todas las señales de entrada y salida del controlador. El enclave solo se deshabilita al presionar el botón de paro (BP), como resultado las señales de entrada y salida se desactivan. 




Figura 5 - Diagrama de conexión eléctrica de los equipos en la estación de control.

En la figura 6 se muestra las conexiones hacia el módulo de salida y en la figura 7 ilustra las conexiones hacia el módulo de entradas digitales 1769-IQ32, localizado en el slot 2. Las conexiones eléctricas de las estaciones neumáticas y almacén se realizaron de forma parecida a la de la estación de control.



Figura 6 - Diagrama de conexión de módulo de salidas de la estación de control. 

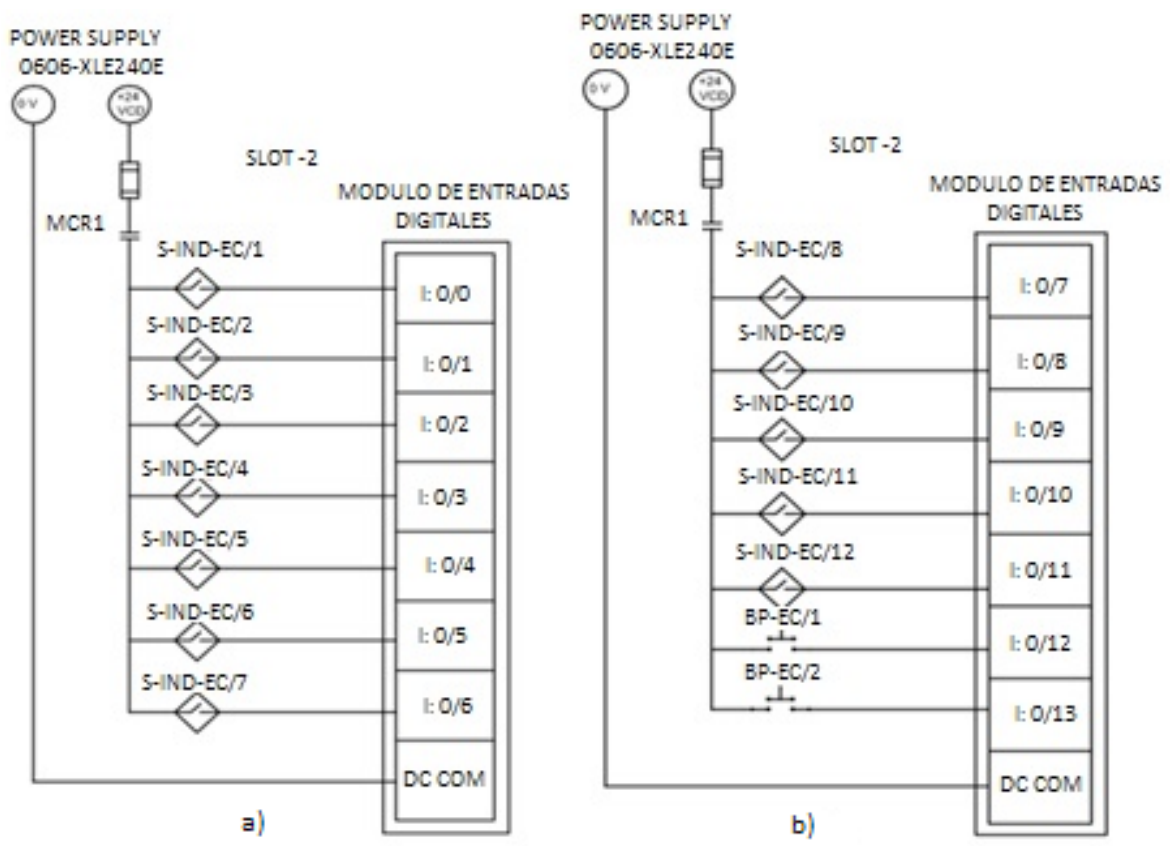

Figura 7 - Diagrama de conexión en los módulos de entrada de la estación de control.

\subsection{Comunicación}

Para conocer que es lo que ocurre en el sistema de manera inmediata cuando esté pasando algún evento, se requiere de un sistema de comunicación que permita la interconexión y trasmisión de datos entre todas las estaciones de trabajo. Para lograr la comunicación fue necesario configurar la red, para lo cual se diseñó una topología de conexión, se realizó la asignación de las direcciones IP a los dispositivos dentro de la red, y se configuro el drive de comunicación. La comunicación de los controladores fue bajo el estándar de Redes de Área local ethernet, es importante mencionar que este protocolo de comunicación fue seleccionado debido a que los controladores PLC se comunican atreves de una red EtherNet/IP (ODVA, 2008).

La topología seleccionada fue en estrella, ya que, en esta, solo se necesita un enlace y un puerto de entrada/salida para conectarse a cualquier número de dispositivos. La Figura 8 muestra el esquema básico de la topología de conexión en estrella utilizada mediante una red Ethernet/IP. La selección de la topología fue el primer paso para la construcción del sistema de conexión entre las estaciones para su comunicación. Para que los dispositivos trabajen dentro de la red Ethernet/IP, se requieren de un numero único e irrepetible de identificación, para asignar esta dirección se hizo uso del programa BOOTP/DHCP Server (Association, 2003), y para establecer la comunicación se utilizó el software RS Linx (Automation, 2008). 




Figura 8 - Topología de conexión en estrella del sistema.

\subsection{Diagrama de flujo y programación}

Una vez realizados los pasos para establecer la red Ethernet/IP, se desarrollaron los diagramas de flujo para la operación de las estaciones de trabajo, y así llevar a cabo la programación con el software RSLogix 5000 (Automation, 2018). En la figura 9 (a), se muestra de forma general el diagrama de flujo de la estación de control. La operación de la estación de control comienza reiniciando las variables presentes en el programa, después esperan la orden de operación; las cuales pueden ser tres. La primera operación se inicia cuando se solicita algún pallet vacío, activándose la "Subrutina de Solicitud de Pallet Vacío"; la segunda operación, contempla enviar una orden hacia la estación de Almacenamiento o a la estación Neumática; la tercera operación comprende el monitoreo de las variables presentes en el CIM-2000. Al finalizar con alguna de las tres operaciones el programa finaliza, pero si se desea continuar con las operaciones el programa puede reiniciar. 



Figura 9 - Diagrama de flujo. a) Secuencia de la estación de control. b) Secuencia de la estación neumática.

Así mismo, en la figura 9 (b), se muestra de forma general el diagrama de flujo de la secuencia de la estación neumática. Esta inicia al activar la estación y enseguida se resetean todas las variables y se escoge el modo de operación; en el "modo automático" las órdenes a ejecutar provendrán de la estación Central de control, en el "modo manual" las operaciones a ejecutar serán introducidas por el operador en la misma estación neumática. Después de establecer el modo de operación se continúa con una solicitud para estacionar un pallet vacío en el puerto de la estación, esta solicitud es enviada a la estación central de control. Posteriormente con la subrutina "alarmas" se asegura de que existan piezas para realizar el pedido, de lo contrario el proceso se pone en pausa hasta que haya piezas disponibles. Se procede con la ejecución de varias subrutinas que culminan con la entrega del pedido solicitado en la banda transportadora; antes de continuar con el siguiente paso se ejecuta una condición que supervisa que se hayan completado todos los pedidos, en caso de no ser así, se repite la secuencia hasta completar todos los pedidos.

En la figura 10, se muestra de forma general el diagrama de flujo de la secuencia de la estación de almacenamiento. 


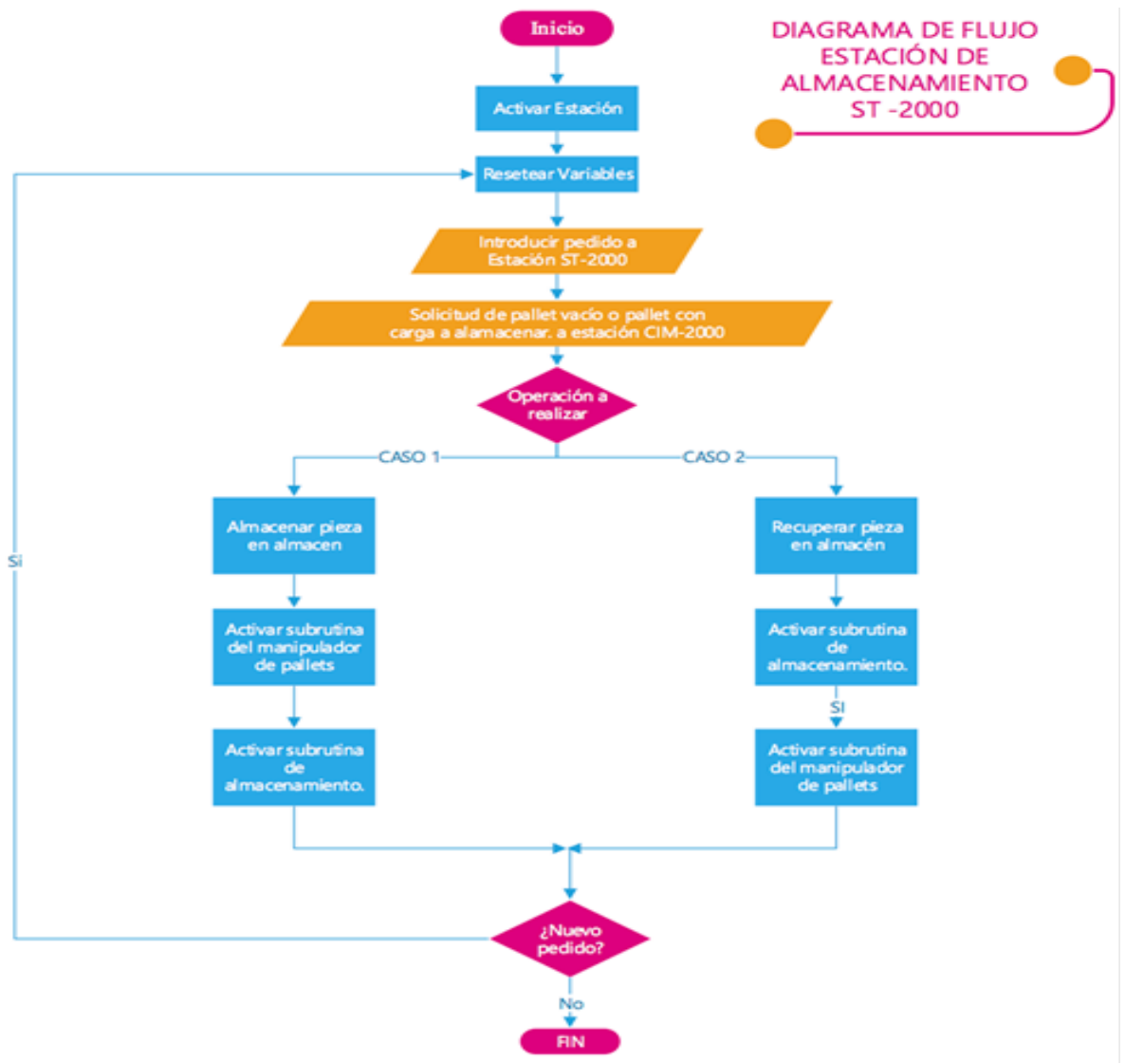

Figura 10 - Diagrama de flujo para la secuencia de la estación de almacenamiento.

La secuencia en la estación de almacenamiento comienza con la activación de la estación, se resetean las variables y se reciben órdenes provenientes de la estación central de control o desde la misma estación de almacenamiento. En caso de que se vaya a recuperar una pieza de almacén, se prosigue con la solicitud para estacionar un pallet vacío en el puerto de la estación; por otro lado, si se almacena una pieza en el almacén, solo se solicita el pallet que contiene la pieza a almacenar. Se prosigue con la cadena de subrutinas que llevan al cumplimiento del pedido. Al término de las secuencias se evalúa si desea un nuevo pedido.

Una vez realizados los diagramas de flujo, se prosiguió a desarrollar los programas en Grafcet en los controladores. En la figura 11 se muestra el programa principal de la estación de control.

\subsection{Pruebas y resultados}

Se establecieron una serie de pruebas para verificar la lógica de programación y así comprobar el funcionamiento de la nueva lógica establecida para el CIM. Entre las 


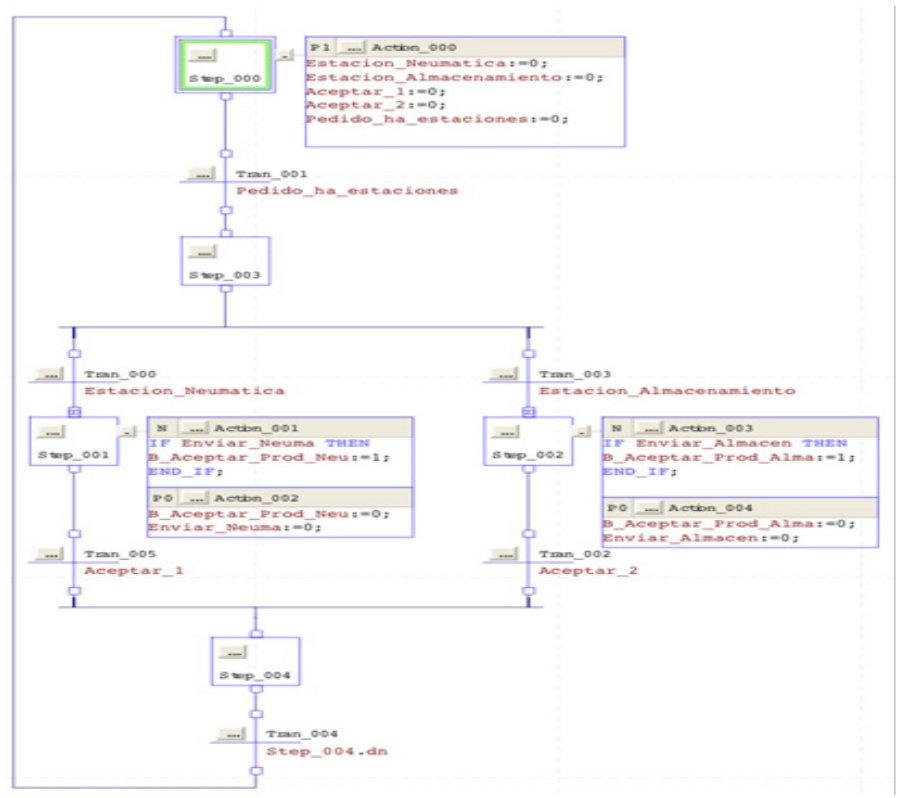

Figura 11 - Programa principal de la estación de control.

pruebas que se realizaron fue la verificación de la conexión física de todos los dispositivos utilizados, comprobando que no hubiera una conexión inadecuada ni falsos contactos. Una vez realizado esto, se prosiguió con las pruebas en el software, verificando que todas las señales de entrada y salida de cada una de las estaciones de trabajo fueran las adecuadas, para después realizar su activación.


Figura 12 - Pruebas de accionamiento de sensores. 
En la figura 12 se observa la prueba de accionamiento de los sensores, donde se ve el funcionamiento del programa y los leds en la tarjeta de salidas del PLC. En la figura 13, se muestra la prueba de comunicación entre estaciones. Una vez realizado esto, se activaron cada una de las estaciones de trabajo por separado, para comprobar que la

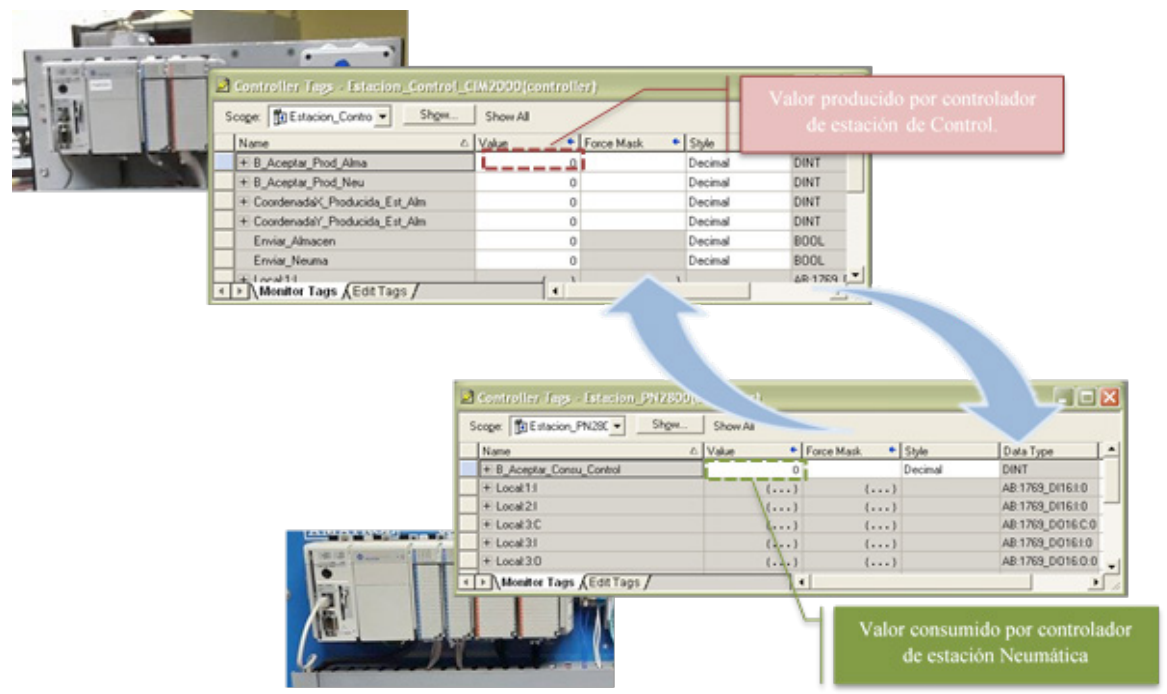

Figura 13 - Pruebas de comunicación entre estaciones.

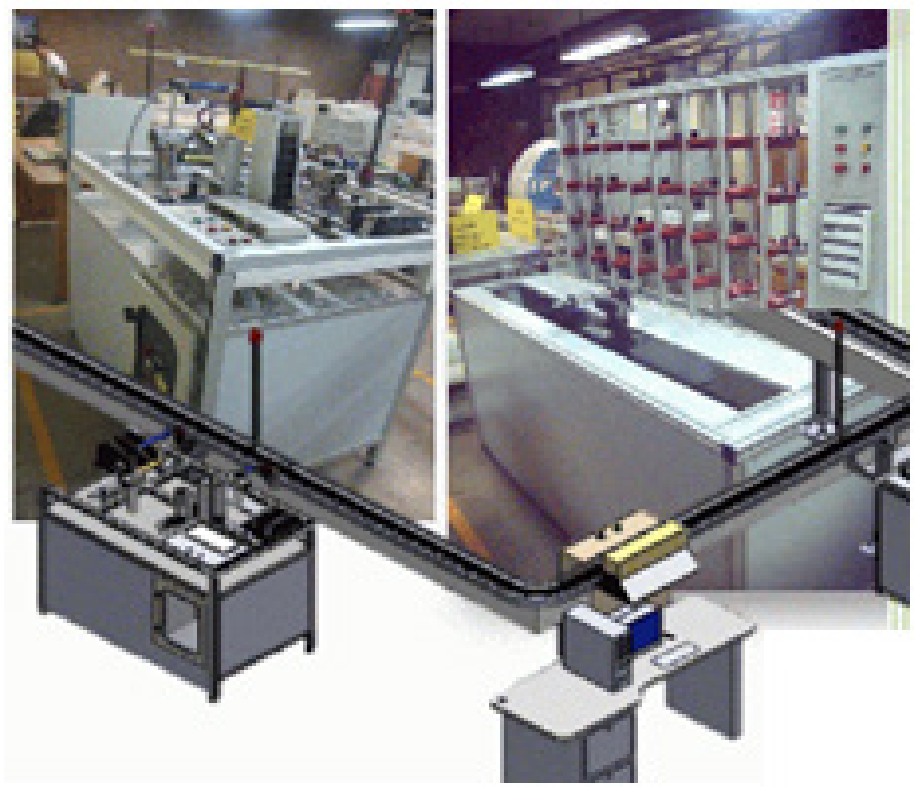

Figura 14 - Integración de las estaciones de trabajo. 
secuencia de operación programada fuera la correcta de acuerdo con la secuencia de establecida. Por último, se realizó la integración de todo el sistema, activando todas las estaciones de trabajo, verificando el adecuado funcionamiento del CIM (Figura 14).

\section{Conclusiones}

El completo rediseño de la instalación eléctrica permite la reducción del cableado necesario para conectar y alimentar todos los dispositivos. Por otra parte, el uso del protocolo Ethernet/IP, sólo necesita un cable para la transmisión de datos, de modo que contribuye a la reducción del cableado.

Los nuevos controladores tienen mejores y más prestaciones en comparación con los viejos PLC que poseía el CIM-2000. Los controladores CompactLogix 5370-L3 y L2 permiten la expansión de los módulos de E/S par futuras ampliaciones del CIM. Estos PLC soportan diversos lenguajes de programación, proporcionando una mayor flexibilidad al momento de programar. También, el uso de datos tipo, productores y consumidores, permite que el intercambio de datos entre los controladores sea fácil y rápida. Además de que permiten una comunicación fluida entre las estaciones.

El cambio del protocolo de comunicación, de Modbus a Ethernet/IP, da mayor aprovechamiento de más funciones. Este protocolo permite el manejo de la información en el tiempo en que aplicaciones discretas son ejecutadas y de proceso continuo, lotes, seguridad, variadores, movimiento y alta disponibilidad, además conecta dispositivos tales como arrancadores de motor y sensores a controladores.

Como trabajo futuro, se propone implementar una interfaz de usuario que permita la supervisión y monitoreo del proceso durante la ejecución de la programación, para que los alumnos puedan entender rápidamente el estado de la máquina y tomen mejores decisiones.

\section{Referencias}

Association, O. D. (2003). Recommended IP Addressing Methods for EtherNet/IP ${ }^{\mathrm{TM}}$ Devices. Ann Arbor, Michigan, United States.

Automation, R. (2008). RSLinx Classic, Cómo Obtener Resultados con RsLinx Classic. Milwaukee, United States.

Automation, R. (2012). Quick Start, CompactLogix 5370 L3 Controllers. Milwaukee, United States.

Automation, R. (2013). Quick Start, CompactLogix 5370 L2 Controllers. Milwaukee, United States.

Automation, R. (2015). Technical Data, 1769 Compact I/O Modules Specifications. Milwaukee, United States.

Automation, R. (2018). Reference Manual, LOGIX 5000 Controllers General Instructions Reference Manual. Milwaukee, United States. 
Elanchezhian, C., Sunder, T., \& Shanmuga, G. (2007). Computer Aided Manufacturing. New Delhi: Laxmi Publications.

García, A., \& Castillo, F. (2007). CIM, el computador en la automatización de la producción. España: Universidad de Castilla-La Mancha.

Grover, M. P., \& Wu, Z. E. (1984). CAD/ CAM: Computer-Aided Desing and Manufacturing. New Jersey: Prentice Hall Inc.

Kalpakjian, S., \& Schmid, S. (2001). Manufacturing Engineering and Technology. U.S.A: Prentice-Hall Inc.

ODVA. (2008). EtherNet/IP Quick Start for Vendors Handbook. Ann Arbor, Míchigan, United States.

Porras, A. (2003). Automatas Programables. México: McGraw Hill.

Radhakrishnan, P., Subramanyan, S., \& Raju, V. (2000). CAD/ CAM / CIM. New Delhi: New Age International.

System, I. T. (1994). Sistema de Entrenamiento del CIM-20oo Mechatronics. Manual de aprendizaje de la estación central de control. México: Degem System. 